\title{
DOI 10.31489/2021No2/56-59
}

UDC 532.5.01

\section{NUMERICAL INVESTIGATION OF THE OCCURRENCE OF A CONCENTRATION-POLARIZATION LAYER}

\author{
Manatbayev R.K., Kalassov N.B., Amankeldi L.B. \\ Al-Farabi Kazakh National University, Almaty, Kazakhstan, kalasov.nurdaulet@gmail.com
}

\begin{abstract}
This work describes the appearance of a concentration polarizing boundary layer on the membrane surface during the separation of the $\mathrm{H}_{2} / \mathrm{CO}_{2}$ gas mixture. Concentration polarization occurs when the rejection solution accumulates near the surface of the membrane, forming a boundary layer. The inclusion of concentration polarization effects in the processing of porous walls creates additional difficulties. The boundary layer formed by concentration polarization can be considered as a type of a second porous wall with a lower permeability than the membrane. The main difficulty in modeling this situation is to determine the appropriate boundary conditions for the concentration on the wall, since the concentrations on the wall will constantly change, and the wall geometry itself may change over time due to particle deposition. To account for this effect, a numerical approach was developed, which is discussed in this work.
\end{abstract}

Keywords: membrane, gas separation, concentration polarization, boundary layer

\section{Introduction}

Biological processes of hydrogen production offer a method by which renewable energy sources, such as biomass, can be used to generate the cleanest energy source for human use [1-2]. The processes of production of combustible gases are promising for the efficient processing of biomass, wastewater treatment in agriculture, public utilities and food production without the release of carbon dioxide into the atmosphere, provided that aerobic and anaerobic bioreactors are combined in a continuous biotechnological scheme [3].

Extraction of energy from the resulting low-pressure gas mixtures is quite problematic, but can be carried out with the help of gas separation technologies specially selected in such a way as to ensure not only low-energy extraction of components, but also complete recirculation of $\mathrm{CO}_{2}$ into the process [4-5].

Biogenic mixtures contain macro-components (methane, hydrogen, and carbon dioxide) and other impurities. The release of energy from such mixtures involves their purification. Despite the high level of development of gas separation technologies, the separation of biological synthesis products by standard methods of chemical technology is economically unprofitable due to the low technical parameters of the resulting liquid and gas mixtures [6-7]. Membrane gas separation can be assigned to the most promising processes for the separation of gas mixtures of biogenic origin. Membrane bioreactors are highly precise, selective, and efficient processes. They can contribute to solving the problems associated with the need to use green and intensive technologies [8].

Concentration polarization occurs when the rejected solution accumulates near the surface of the membrane, forming a boundary layer. This accumulation of solute causes a diffusive flow back to the source material, which ultimately reaches a stationary state in which the flow of solute to the membrane surface equals the solute flux through the membrane plus the diffusive flow from the membrane surface back to the source material. The establishment of this boundary layer ensures the subsequent contamination of the membrane layer; thus, the minimization or removal of the concentration polarization layer is the main goal of the membrane study.

In this paper, based on the results of hydrodynamic modeling of membrane separation of gases $\mathrm{H}_{2}$ / $\mathrm{CO}_{2}$ [9], an analytical method for the emergence of a concentration polarization boundary layer is described. The basic hydrodynamic model was tested on the basis of experimental and computational data from the literature [10], and the strategy and modeling methods typical for the problem of membrane separation were discussed. 


\section{Analytical method for determining the mass coefficient}

The film theory approach for describing concentration polarization was developed by Michaels et al [11-12]. The film theory simplifies the complex transfer problem to a one-dimensional mass transfer problem by assuming that the axial convection of the solute near the membrane surface is insignificant. Integrating the one-dimensional (transverse) balance of the convective-diffusion mass from the membrane surface to the thickness of the boundary layer (film) of the final mass $\delta$, we obtain the ratio between the concentration polarization and the permeate flow.

$$
\frac{C_{w}-C_{p}}{C_{b}-C_{p}}=\exp \left(\frac{v_{w} \delta}{D}\right)
$$

where $C_{w}$ - the concentration on the surface of the membrane for the rejected $\mathrm{CO}_{2}, C_{b}$ and $C_{p^{-}}$volume concentration and solute concentration, respectively, $v_{w}$ is the rate of penetration of $\mathrm{H}_{2}$ on the channel wall, and $\mathrm{D}$ - the diffusion coefficient of the solute.

The procedure for estimating the thickness of the convective-diffusion layer in a channel with instant or promptly reacting walls is the basis of most models of the film theory. For a fully developed laminar current in a thin rectangular channel, the thickness of the film layer is described

$$
\frac{\delta(x)}{x}=1.475\left(\frac{h}{x}\right)^{2 / 3}\left(\frac{D}{u_{\max } * h}\right)^{1 / 3}
$$

where $x$ - the longitudinal coordinate, $\mathrm{h}$ - half the height of the channel, and $u_{\text {max }}$-the maximum crossflow velocity at the center of the channel. Assuming a constant diffusion coefficient, the local mass transfer coefficient is described by

$$
k(x)=\frac{D}{\delta(x)}=\frac{1}{1.475}\left(\frac{3 \bar{u} D^{2}}{2 h x}\right)^{1 / 3}=0.538\left(\frac{\dot{\gamma}_{w} D^{2}}{x}\right)^{1 / 3},
$$

where $\bar{u}$ - the average volume velocity of the cross-flow and $\dot{\gamma}_{w}=\frac{3 \bar{u}}{h}$-the shear rate of the wall. The main limitation of using film theory to membrane separation is the assumption that the transversal component of convection (permeate flow) does not affect the thickness of the boundary layer, $\delta$.

The channel-averaged mass transfer coefficient $\mathrm{k}$ is obtained from the integration of the local flow $v_{w}(\mathrm{x})$ determined by equations (2) and (3), provided that the permeate and volume concentration, as well as the permeate and cross-flow velocities, remain constant along the length of the membrane channel, $\mathrm{L}$. This assumption is unrealistic for large-scale reverse osmosis systems, but is sensible for short membrane channels where recovery is insignificant. The average channel transfer mass coefficient is expressed as

$$
\bar{k}=0.807\left(\frac{\dot{Y}_{w} D^{2}}{L}\right)^{1 / 3},
$$

which is matching to the mass transfer correlation for a laminar flow in a slim channel, usually represented as a Sherwood number,

$$
S h=\frac{\bar{k} d_{H}}{D}=1.85\left(\operatorname{ReScd}_{H} / L\right)^{1 / 3}
$$

\section{Results and discussion}

Figure 1 shows the dependence of the tangential voltage on the number Re at the channel input. As can be seen from the resulting graph, the tangential voltage increases with an increase in the speed at the entry to the channel. This confirms that a higher Reynolds number at the input corresponds to a thinner boundary layer of concentration polarization.

By the analytical method, using the hydrodynamic results of equation (4), the average mass transfer coefficient along the length of the channel was calculated. Knowing the average value of the mass transfer coefficient for the corresponding Reynolds number, the Sherwood number was calculated. Figure 2 shows the dependence of the Sherwood number on the Reynolds number. Also, in Figure 3, the change in the mass transfer coefficient along the length of the channel was derived. 


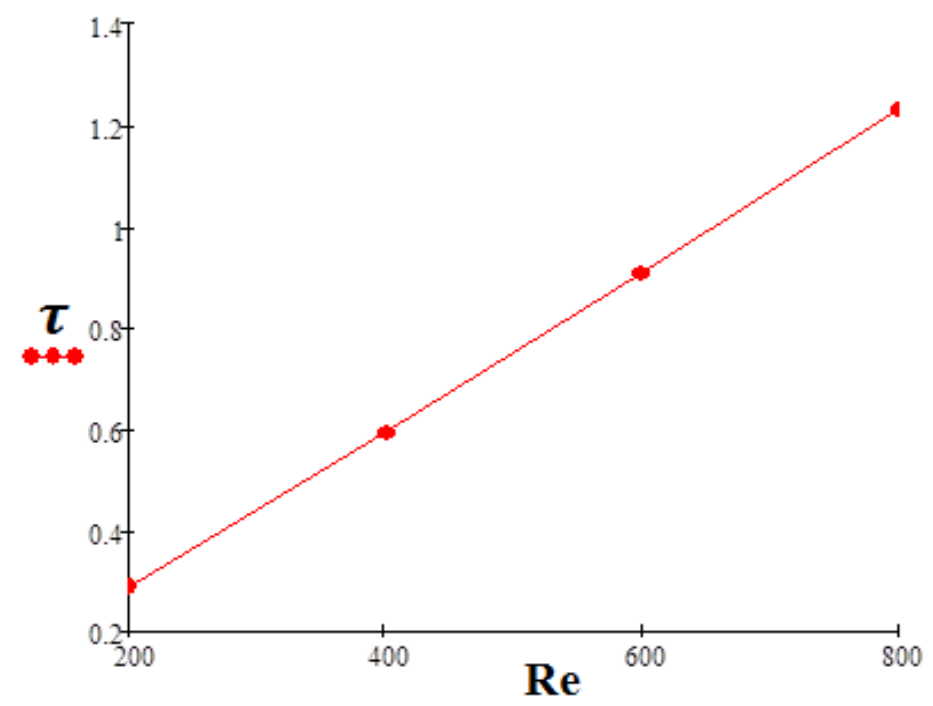

Fig.1. Dependence of the tangential voltage on the number Re at the channel input.

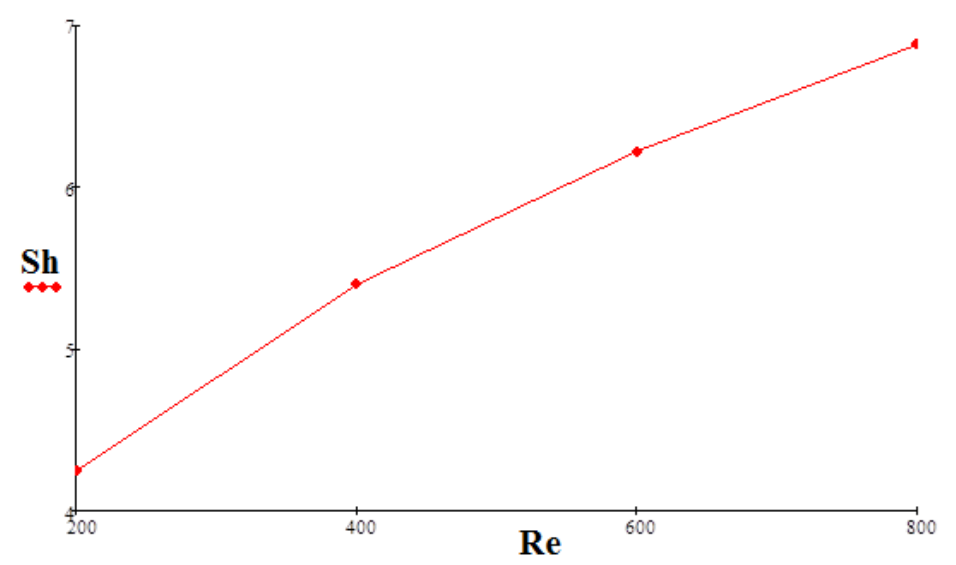

Fig.2. Dependence of the Sherwood number on the Reynolds number.

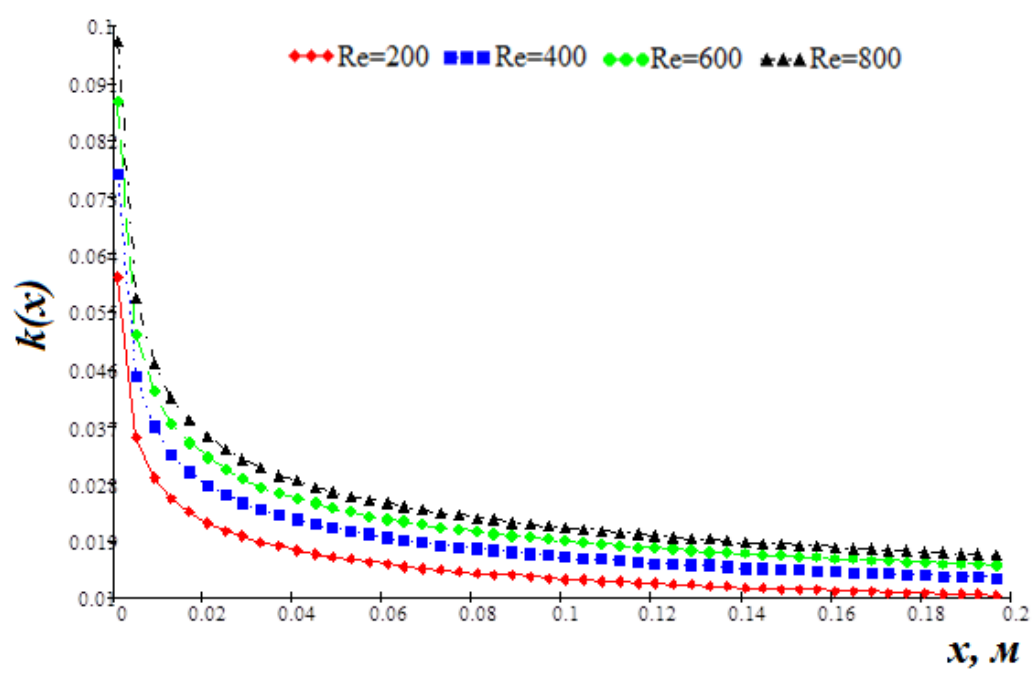

Fig.3. Change in the mass transfer coefficient along the length of the channel. 


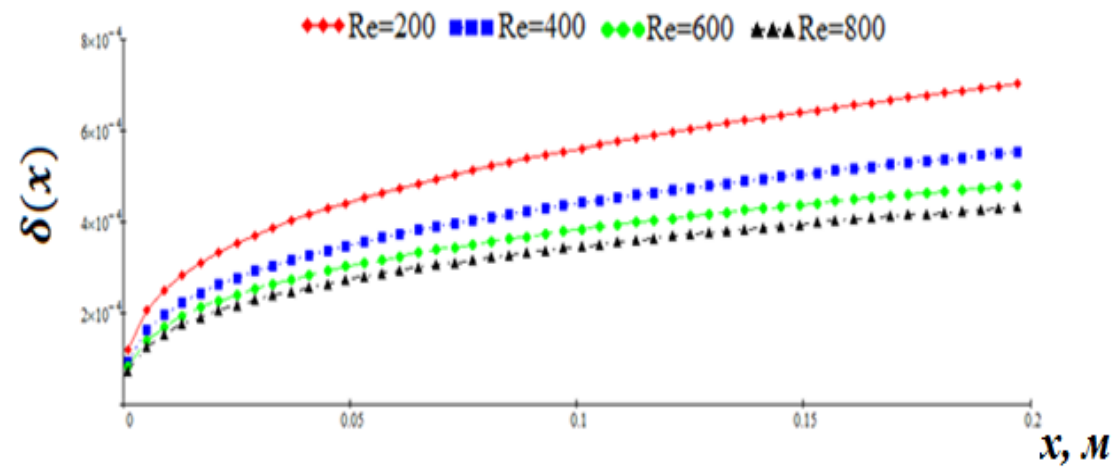

Fig.4. The change in the thickness of the concentration polarization boundary layer along the channel length.

As can be seen in Figure 4, the thickness of the concentration polarization layer increases along the length of the channel. As expected, a higher Reynolds number at the input corresponds to a thinner boundary layer of concentration polarization due to an increased shift that disrupts the boundary layer of concentration polarization.

\section{Conclusion}

The inclusion of concentration polarization effects in the treatment of porous membrane walls creates additional difficulties. As expected, a thinner boundary layer of concentration polarization due to an increase in the shift that violates the boundary layer of concentration polarization.Comparison with analytical, experimental and computational data shows that the modern approach to CFD modeling is capable of describing flow conditions for 2D membrane channels. Good agreement is observed from the point of view of the formation of a concentration polarization boundary layer. This gives confidence that the basic hydrodynamic model of the membrane filtration process developed in [9] is satisfactory.

\section{REFERENCES}

1 Akinbomi J., Wikandari R., et al. Enhanced fermentative hydrogen and methane production from an inhibitory fruit-flavored medium with membrane capsulated cells. Membranes.2015, Vol.5, No. 4, pp. 616-631.

2 Kalassov N.B., Dzhonova D., TsibranskaI., et al. Application of integrated membrane bioreactors in renewable energy industry. Journal of Chemical Technology and Metallurgy. 2020, Vol. 55, No. 2, pp. 314-323.

3 Adekunle K.F., Okolie J.A. A review of biochemical process of anaerobic digestion. Adv. Biosci. Biotechnol. 2015, Vol. 6, No. 3, pp. 205-207.

4 Bakonyi P., et al. Biohydrogen purification using a commercial polyimide membrane module: studying the effects of some process variables. Int. J. Hydrogen Energy. 2013, Vol. 38, No. 35, pp. 15092-15099.

5 Bakonyi P., Nemestóthy N., Bélafi-Bakó K. Biohydrogen purification by membranes: an overview on the operational conditions affecting the performance of nonporous, polymeric and ionic liquid based gas separation membranes. Int. J. Hydrogen Energy. 2013, Vol. 38, No, 23, pp. 9673-9687.

6 Ghimire A., Kumar G., Sivagurunathan P., et al. Bio-hythane production from microalgae biomass: key challenges and potential opportunities for algal bio-refineries. Bioresour. Technol. 2017, Vol. 241, pp. 525-536.

7 Ghimire A., Frunzo L., Pirozzi F., et al. A review on dark fermentative biohydrogen production from organic biomass: process parameters and use of by-products. Appl. Energy. 2015, Vol. 144, 73-95.

8 Rosalinda M., Loredana D.B., Enrico D. Membrane Bioreactors for Production and Separation. Comprehensive Biotechnology. 2019, Vol. 2, pp. 374-393.

9 Kalassov N.B., Dzhonova D., Manatbayev R.K. Hydrodynamic modeling of the cross-flow of membrane separation processes. Bulletin» of the national engineering academy of the republic of Kazakhstan. 2020, Vol. 75, No.1, pp. 68-76.

10 Geraldes V., Semião V., De Pinho M.N. Flow and mass transfer modelling of nanofiltration. J. membrane sci. 2001, Vol. 191, 109-128.

11 Michaels A.S. New separation technique for the CPI. Chem. Eng. Prog. 1968, Vol. 64, pp. 31-33.

12 Porter M.C., Concentration polarization with mem-brane ultrafiltration, Ind. Eng. Chem. Prod. Res. Develop. 1972, Vol. 11, pp. 234-237. 